Geschlechtervergleich

\title{
Frauen mit Vorhofflimmern trifft häufiger ein Insult
}

\author{
Frauen mit Vorhofflimmern verspüren häufiger Symptome als Männer, \\ und auch die Lebensqualität scheint deutlicher beeinträchtigt. \\ Paradox: Die Rhythmusstörung zieht bei weiblichen Patienten häufiger \\ einen Schlaganfall nach sich, führt aber seltener zum Tod.
}

_ Dass es bei Vorhofflimmern (VHF) deutliche Unterschiede zwischen den Geschlechtern gibt, hat ein Team der Duke University (Durham) in einer prospektiven Multicenter-Studie gezeigt. An dieser waren 10.135 Patienten mit elektrokardiografisch bestätigtem VHF beteiligt (51\% paroxysmal, $17 \%$ persistierend, $28 \%$ permanent, der Rest neu entdeckt), darunter 4.293 Frauen.

\section{Bei Frauen öfter symptomatisch}

Unter Palpitationen litten 40\% der Frauen, aber nur 27\% der Männer. Der Anteil der Patientinnen mit Schwindel betrug $23 \%$ (vs. $19 \%$ ); $28 \%$ (vs. $25 \%$ ) berichteten über Fatigue. Wie Dr. Jonathan P. Piccini vom Duke Center for Atrial Fibrillation und sein Team berichten, war nur knapp jede dritte Frau mit VHF $(32,1 \%)$ asymptomatisch, bei den Männern waren es $42,5 \%$.

Die Lebensqualität hatten die Forscher in einer Substudie mit 2.007 Patienten untersucht. Als Maßstab diente der AFEQT-Score (Atrial Fibrillation Effects on Quality of Life). Auf der 100-Punkte-Skala (100 beim bestmöglichen Zustand) erreichten die Frauen im Schnitt einen Wert von 80, die Männer von $83(\mathrm{p}<0,001)$. Der Unterschied blieb auch dann signifikant, wenn man nur Patienten ab dem 75. Lebensjahr berücksichtigte, und bestand auch bei einer Nachbeobachtung über zwei Jahre fort.

\section{Unterschiede bei der Therapie}

Bei der Einnahme von Antiarrhythmika lagen beide Geschlechter gleichauf (28,9\% vs. $28,6 \%)$, ebenso bei der Kathe- terablation des VHF. Für Piccini et al. erstaunlich: Die Patientinnen hatten etwa doppelt so oft eine Ablation des AV-Knotens erhalten $(2,9 \%$ vs. $1,7 \%$; HR $=1,97)$. Diese Methode gilt als Ultima Ratio bei therapierefraktärem Vorhofflimmern. Nur bei 25,8\% der Frauen lag den Autoren zufolge ein permanentes VHF vor (Männer: 29,5\%). Der Anteil paroxysmaler bzw. persistierender Formen war bei beiden Geschlechtern in etwa gleich.

Bei Betablockern und Aspirin hatte man bei den Frauen etwas mehr gespart (62\% und $21,5 \%$ gegenüber $65,5 \%$ und $30,9 \%)$, dafür hatten diese etwas mehr Digoxin erhalten (24,6\% vs. $22,6 \%$ ).

\section{Niedrigere Mortalität}

Nach einem mittleren Beobachtungszeitraum von 2,3 Jahren hatten die Frauen in der bereinigten Analyse ein deutlich geringeres Risiko für fatale Ereignisse: So lag die Gesamtmortalität um 43\% und die kardiovaskuläre Mortalität um 44\% niedriger als bei den Männern. Allerdings fanden die Forscher keinen nennenswerten Unterschied bei neu aufgetretener Herzinsuffizienz, Myokardinfarkt, großen Blutungen und plötzlichem Herztod. Beide Geschlechter wurden zudem in etwa gleich häufig revaskularisiert und stationär eingewiesen.

\section{0\% höheres Schlaganfallrisiko}

Überraschend ist vor diesem Hintergrund: Sowohl in der unbereinigten als auch in der bereinigten Auswertung waren das Schlaganfallrisiko und das Risiko für Embolien außerhalb des ZNS für Frauen signifikant höher (HR 1,39).
Weitere Studien müssen nun klären, womit das Paradoxon des um nahezu $40 \%$ erhöhten Schlaganfallrisikos bei deutlich geringerer Mortalität bei den Frauen zusammenhängt. Denkbar wäre, dass therapeutische Strategien zur Frequenz- und Rhythmuskontrolle das Fortschreiten der Erkrankung beeinflussen. Auch Depressionen wurden in früheren Studien mit der erhöhten Krankheitslast bei VHF assoziiert.

Der Unterschied in der Lebensqualität - immerhin 24\% in der adjustierten Analyse - könnte, so Piccini, sowohl physisch als auch psychisch bedingt sein. Die Studie habe gezeigt, dass Frauen in beiderlei Hinsicht stärker unter der Rhythmusstörung litten als Männer. -

Dr. Elke Oberhofer

- Piccini JP et al. Differences in Clinical and Functional Outcomes of Atrial Fibrillation inWomen and Men: Two-Year Results From the ORBIT-AF Registry. JAMA Cardiol 2016, online 18. Mai

\section{FAZIT FÜR DIE PRAXIS}

1. Die Frauen waren im Schnitt häufiger symptomatisch und in ihren Alltagsaktivitäten beeinträchtigt und hatten eine schlechtere Lebensqualität als die männlichen Teilnehmer.

2. Weibliche Patienten erhielten häufiger als männliche eine AV-Knoten-Ablation.

3. Das Risiko, einen Schlaganfall oder eine Embolie außerhalb des ZNS zu erleiden, war bei den Frauen höher.

4. Die Gesamtüberlebensrate im Beobachtungszeitraum war bei den Frauen höher und das Risiko für einen kardiovaskulären Tod geringer als bei den Männern. 